PHYSICAL REVIEW A 81, 043817 (2010)

\title{
Bichromatic emission and multimode dynamics in bidirectional ring lasers
}

\author{
Antonio Pérez-Serrano* \\ Instituto de Física Interdisciplinar y Sistemas Complejos, IFISC, UIB-CSIC, Campus UIB, Palma de Mallorca, E-07122, Spain \\ Julien Javaloyes \\ Department of Electronics and Electrical Engineering, University of Glasgow, Glasgow, G12 8LT, United Kingdom \\ Salvador Balle \\ Institut Mediterrani d'Estudis Avançats, IMEDEA, UIB-CSIC, Esporles, E-07190, Spain
}

(Received 9 October 2009; published 15 April 2010)

\begin{abstract}
The multimode dynamics of a two-level ring laser is explored numerically using a bidirectional traveling wave model retaining the spatial effects due to the presence of counter-propagating electric fields in the population inversion. Dynamical regimes where the emission in each direction occurs at different wavelengths are studied. Mode-locked unidirectional emission for large gain bandwidth and relatively small detuning is reported.
\end{abstract}

DOI: 10.1103/PhysRevA.81.043817

PACS number(s): 42.55.Wd, 42.60.Da, 42.79.Gn

\section{INTRODUCTION}

Since their inception, ring lasers (RLs) have attracted the interest of both theoretical and experimental research. The design of the RL cavity has a high degree of rotational symmetry with very low-ideally vanishing-backscattering of waves propagating in one direction and the opposite. Moreover, it opens the possibility of tailoring the reversibility of the optical paths and impose unidirectional lasing by introducing extra losses to one of the propagation directions. This allows to more efficiently extract power from these devices, hence the unidirectional RL design is at the heart of many high-power solid state and fiber lasers [1-3], which are commonplace in many applications.

From the theoretical point of view, the reversibility of the optical path and the absence of reflectivity allow for two degenerate counter-propagating electric fields in the same gain medium. This property - which is not exclusive of RLs [4], but common to all systems possessing rotational symmetry as, for example, micro-disk lasers [5,6]—has important potential applications. A possible state of bidirectional emission has immediate potential applications for the development of laser gyroscopes [7], but the strong competition for the gain among these counter-propagating states should lead to bistable unidirectional operation [8], which can also be exploited for all-optical signal processing and storage [9]. However, the first systematic formulation of the theory of RLs by Lamb et al. [10-12] already evidenced that symmetry issues and even minute intra-cavity reflections have a major impact on the modal structure in RLs: pure counter-propagating traveling waves are ideal states only allowed in closed-loop optical cavities without any localized reflection; localized reflections destroy the rotational invariance of the RL, and the cavity modes become nondegenerate standing waves. These effects, together with the nonlinear interaction of the counter-propagating waves mediated by the active medium, lead to a large variety of operating regimes and dynamics that are profoundly different from those of Fabry-Pérot lasers

*antonio@ifisc.uib-csic.es
[13-17] and that have posed problems for the development of practical devices (specially semiconductor-based) for the above applications in spite of the tremendous advances achieved in the processing and technology [18-25].

The above phenomena have pointed out the necessity of proper models for RLs that are able to describe the different regimes of operation of these devices. RLs of different types are often modeled by rate equations (REs) [14,26-28]. These models, based on a $\chi_{3}$ description of the polarization induced in the active medium, usually describe the temporal evolution of the amplitude (or power) of the modes of the electric field and the material gain without taking into account the spatial effects explicitly. Instead-since the spatially varying field profiles induce inhomogeneities in the carrier density that couple the field modes-phenomenological mode-coupling coefficients are used to describe different processes of modal competition. The main advantage of such models is that they provide an intuitive picture of the mechanisms underlying mode dynamics, and they have allowed one to successfully fit the emission spectra under different operation conditions, hereby permitting one to determine several important device parameters. Nevertheless, one of the main difficulties encountered in this type of model is the large number of parameters required to describe the different nonlinear mechanisms of modal coupling-such as carrier density pulsations, spatial and spectral hole burning, and carrier heating. In addition, one has to determine a priori which modes have to be accounted for in the modeling: increasing the number of modes allows one in principle to improve the dynamical description of the system, but at the same time more mode-coupling parameters have to be determined.

A natural way to take into account the spatial effects and describe multimode dynamics is to use a traveling wave model (TWM). A TWM is a semiclassical description of the system that allows one to describe any kind of resonator by including the appropriate boundary conditions, and it has already been used for Fabry-Pérot lasers [29,30], for unidirectional RLs [31], and even for bidirectional RLs [32]. The TWM offers a comprehensive model of the RL, but adds a bigger computational cost, because RE models are based in systems of ordinary differential equations (ODEs) and a TWM 
description is based in systems of partial differential equations (PDEs). In this work, we study the multimode dynamics of RLs by means of a bidirectional TWM that preserves the spatial variations of the field amplitudes and that explicitly considers the dominant contribution of the spatial grating in the gain, which are often disregarded. Since our goal is to formulate a description of the RL allowing for bidirectional emission and mode competition that retains the essential features of the dynamics, we consider a simple two-level active medium. In this case, the interaction of the active medium and the optical field is well known and its description in time domain poses no additional problems as would be the case, for example, for semiconductor systems. In addition, although a complete description of the system requires the full three-dimensional wave equation to describe the cavity field exactly, our approach only takes into account the axial direction of propagation by assuming a single-transverse mode be supported by the cavity.

The paper is organized as follows. In Sec. II, the TWM is summarized, with the detailed derivation of the model and its numerical implementation in the appendices. The laser threshold and modes are analytically or semianalytically determined. In Sec. III, we test the correctness and accuracy of the numerical algorithm used to implement the TWM by comparing the results obtained in the single-mode limit with those in [33]. In Sec. IV, the multimode dynamics of the system are discussed, focusing on two cases: moderate and large gain bandwidth. We find dynamical regimes where the emission in each direction is almost single mode, but each direction lases at a different wavelength. This regime might have practical relevance in the development of gyroscopes, since the two directions are not frequency locked. We also find regimes of mode-locked unidirectional emission for large gain bandwidth and relatively small detuning. Finally, the conclusions are presented in Sec. V.

\section{THE MODEL}

In this section we briefly summarize the traveling wave model (TWM) considered for the ring laser. The details of its derivation can be found in Appendix A.

We consider that the electric field is quasimonochromatic and it is decomposed into forward (+) and backward (-) waves propagating in opposite directions. The active medium is assumed to be composed by homogeneously broadened two-level atoms. We also consider a quasiresonant light matter interaction in the rotating wave approximation (RWA). The population inversion density, $D$, is decomposed in different spatial harmonics of a fundamental modulation at half the optical wavelength and, in the resulting hierarchy of contributions, we retain the first-order term.

With these assumptions, the dimensionless equations that define the TWM read

$$
\begin{aligned}
\pm & \frac{\partial A_{ \pm}}{\partial s}+\frac{\partial A_{ \pm}}{\partial \tau}=B_{ \pm}-\alpha A_{ \pm}, \\
\frac{1}{\gamma} \frac{\partial B_{ \pm}}{\partial \tau}= & -(1+i \tilde{\delta}) B_{ \pm}+g\left(D_{0} A_{ \pm}+D_{ \pm 2} A_{\mp}\right) \\
& +\sqrt{\beta D_{0}} \xi_{ \pm}(s, \tau)
\end{aligned}
$$

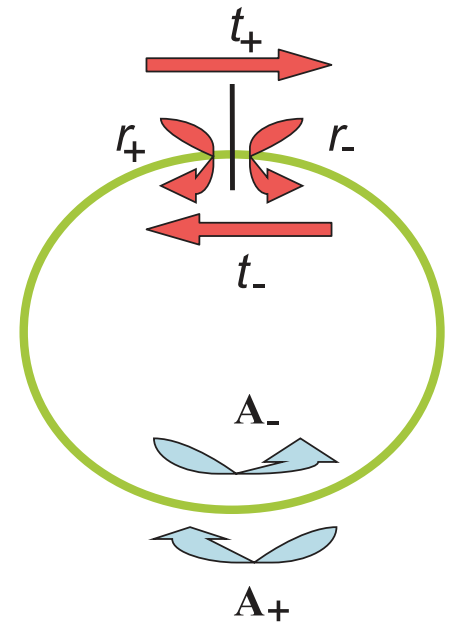

FIG. 1. (Color online) Schematic representation of the ring laser boundary conditions where $t_{ \pm}$and $r_{ \pm}$are the reflectivity and transmissivity for the counter-propagating fields $A+$ and $A_{-}$, respectively.

$$
\begin{gathered}
\frac{1}{\epsilon} \frac{\partial D_{0}}{\partial \tau}=J-D_{0}+\Delta \frac{\partial^{2} D_{0}}{\partial s^{2}}-\left(A_{+} B_{+}^{*}+A_{-} B_{-}^{*}+\text { c.c. }\right) \\
\frac{1}{\eta} \frac{\partial D_{ \pm 2}}{\partial \tau}=-D_{ \pm 2}-\frac{\epsilon}{\eta}\left(A_{ \pm} B_{\mp}^{*}+A_{\mp}^{*} B_{ \pm}\right)
\end{gathered}
$$

where $A_{ \pm}$are the slowly varying components of the counterpropagating electric fields, $B_{ \pm}$are their respective polarizations, $D_{0}$ is the quasihomogeneous inversion density, and $D_{ \pm 2}$ are the spatially dependent contributions to the grating in the population inversion density, $\alpha$ are the internal losses, $\widetilde{\delta}$ is the detuning, $\epsilon$ and $\eta$ are the decay times for $D_{0}$ and $D_{ \pm 2}$, respectively, and $\gamma$ determines the spectral width of the gain spectrum. For more details see Appendix A.

Equations (1)-(4) must be completed with the boundary conditions for the electric fields. We consider the most general case depicted in Fig. 1, hence the boundary conditions read

$$
\begin{gathered}
A_{+}(0)=t_{+} A_{+}(1) e^{i \gamma \widetilde{\omega}_{0}}+r_{-} A_{-}(0), \\
A_{-}(1) e^{-i \gamma \widetilde{\omega}_{0}}=t_{-} A_{-}(0)+r_{+} A_{+}(1) e^{i \gamma \widetilde{\omega}_{0}},
\end{gathered}
$$

where $r_{ \pm}$and $t_{ \pm}$denote the reflectivity and transmissivity of the forward and backward waves. These coefficients can in general be different for the two directions in order to describe the effect of nonreciprocal elements as an optical isolator. We note, moreover, that $\left|t_{ \pm}\right|^{2}+\left|r_{ \pm}\right|^{2}=1-\varepsilon_{ \pm}$, where $\varepsilon_{ \pm}$are the losses at the point coupler.

These general boundary conditions reduce to those for an ideal ring if $r_{ \pm}=0$ and $t_{ \pm} \neq 0$, and to those for a Fabry-Pérot cavity if $r_{ \pm} \neq 0$ and $t_{ \pm}=0$. When $r_{+}=r_{-}$and $t_{+}=t_{-}$the device is symmetrical for the two propagation directions.

In the following we shall take $\gamma \widetilde{\omega}_{0}=2 \pi m$ where $m=$ $0, \pm 1, \pm 2 \ldots$ then $e^{i \gamma \widetilde{\omega}_{0}}=1$ without loss of generality: it simply means that we take as the carrier frequency $\omega_{0}$ that is corresponding to one of the modes of the cavity. Moreover, we shall restrict ourselves to symmetric devices unless explicitly noted. 


\section{A. Laser threshold}

The lasing threshold of the system can be readily determined by performing the linear stability analysis (LSA) around the off solution (i.e., $A_{ \pm}^{\text {st }}=0, B_{ \pm}^{\text {st }}=0, D_{ \pm 2}^{\text {st }}=0$, and $D_{0}^{\text {st }}=J$. We linearize (1)-(4) around this solution by introducing the small perturbations,

$$
\begin{gathered}
A_{ \pm}=\varepsilon a_{ \pm}(s, \tau), \quad B_{ \pm}=\varepsilon b_{ \pm}(s, \tau), \\
D_{0}=J+\varepsilon^{2} d_{0}(s, \tau), \quad D_{ \pm 2}=\varepsilon^{2} d_{ \pm 2}(s, \tau) .
\end{gathered}
$$

Where $\varepsilon$ is infinitesimally small, then retaining the terms to first order in $\varepsilon$ and assuming that the perturbations evolve in time as

$$
a_{ \pm}(s, \tau)=\widetilde{a}_{ \pm}(s) e^{\lambda \tau}, \quad b_{ \pm}(s, \tau)=\widetilde{b}_{ \pm}(s) e^{\lambda \tau},
$$

we can obtain the eigenvalues $\lambda_{m}(m=0, \pm 1, \pm 2, \ldots)$ whose real part determines whether or not the mode $m$ is stable and whose imaginary part determines the modal frequency. The modal threshold is thus given by the current value $J_{m}^{\text {th }}$ such that $\operatorname{Re}\left(\lambda_{\mathrm{m}}\right)=0$. In our case, we obtain two different branches of solutions $(\sigma= \pm 1)$ whose modal thresholds read

$$
J_{m}^{\mathrm{th}}(\sigma)=\frac{(\gamma \tilde{\delta}-2 \pi m)^{2}\left(\alpha+\ln \frac{1}{t+\sigma r}\right)}{g\left(\alpha+\gamma+\ln \frac{1}{t+\sigma r}\right)^{2}}+\frac{1}{g} \ln \frac{1}{t+\sigma r},
$$

and which have modal frequencies,

$$
\Omega_{m}(\sigma)=\frac{2 \pi m+\widetilde{\delta}\left(\alpha+\ln \frac{1}{t+\sigma r}\right)}{1+\frac{1}{\gamma}\left(\alpha+\ln \frac{1}{t+\sigma r}\right)} .
$$

The thresholds for the two branches of solutions are shown in Fig. 2 for typical ring laser parameters. For the small modal index, the Lorentzian can be approximated by a parabola, which explains the shape of Fig. 2, and the minimum threshold corresponds to the gain peak. The two branches of solutions arise from the nonvanishing reflectivity $r$ : when $r=0$, the modes are pure forward and backward waves which are degenerate; however, for $r \neq 0$ the rotational invariance of the system is broken and the modes are given by combinations of the forward and backward waves that lift this degeneracy

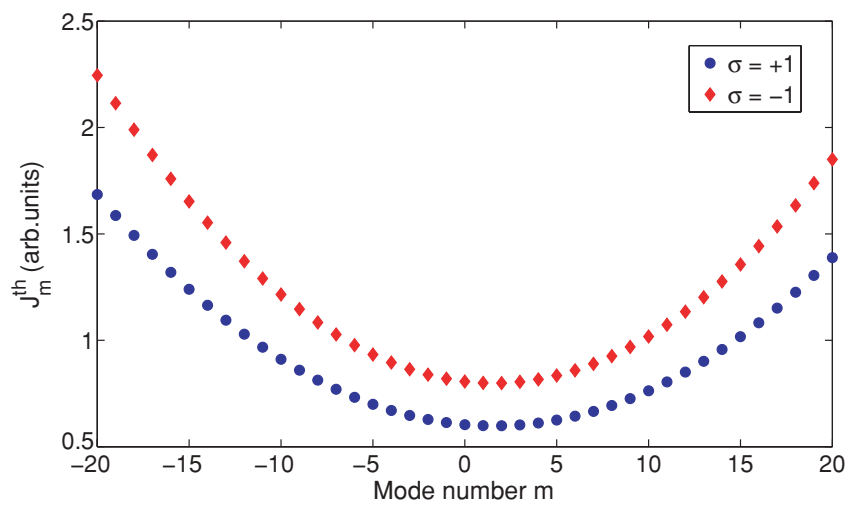

FIG. 2. (Color online) $J_{m}^{\text {th }}$ versus $m . \widetilde{\delta}=0.1, g=1, t=0.5$, $r=0.05, \alpha=0$, and $\gamma=100$. In this case, the lowest threshold corresponds to mode $m=2$ with $J_{2}^{\text {th }}=0.5981$ for $\sigma=+1$. in both frequency and threshold gain. For $r \rightarrow 0$, Eqs. (8) and (9) read

$$
\begin{aligned}
J_{m}^{\mathrm{th}}(\sigma)= & \frac{(\gamma \widetilde{\delta}-2 \pi m)^{2}(\alpha-\ln t)}{g(\alpha+\gamma-\ln t)^{2}}-\frac{1}{g} \ln t \\
& +\frac{\sigma(\gamma \tilde{\delta}-2 \pi m)^{2}(\alpha-\gamma-\ln t)}{g t(\alpha+\gamma-\ln t)^{3}} r+O(r)^{2},
\end{aligned}
$$

and

$$
\begin{aligned}
\Omega_{m}(\sigma)= & \frac{\gamma[2 \pi m+\tilde{\delta}(\alpha-\ln t)]}{\alpha+\gamma-\ln t} \\
& +\frac{\gamma \sigma(2 \pi m-\gamma \delta)}{t(\alpha+\gamma-\ln t)^{2}} r+O(r)^{2} .
\end{aligned}
$$

Such an effect has been experimentally observed in semiconductor ring lasers [34] where the residual reflectivities in the laser cavity induced modal doublets that correspond to the mode-pulling formula (9). The threshold difference for these doublets is roughly proportional to $r$ for small reflectivities hence the gain difference can be hardly noticeable specially for appreciable internal losses $\alpha$.

\section{B. Monochromatic solutions}

The nontrivial monochromatic solutions read

$$
\begin{gathered}
A_{ \pm}=A_{ \pm}^{\mathrm{st}} e^{-i \widetilde{\omega} \tau}, \quad B_{ \pm}=B_{ \pm}^{\mathrm{st}} e^{-i \widetilde{\omega} \tau}, \\
D_{0}=D_{0}^{\mathrm{st}}, \quad D_{ \pm 2}=D_{ \pm 2}^{\mathrm{st}},
\end{gathered}
$$

where $\widetilde{\omega}$ is the lasing frequency. We use (12) in (1)-(4) finding

$$
\begin{gathered}
\pm \frac{\partial A_{ \pm}^{\mathrm{st}}}{\partial s}+(\alpha-i \widetilde{\omega}) A_{ \pm}^{\mathrm{st}}=B_{ \pm}^{\mathrm{st}} \\
B_{ \pm}^{\mathrm{st}}=\frac{g=\left(D_{0}^{\mathrm{st}} A_{ \pm}^{\mathrm{st}}+D_{ \pm 2}^{\mathrm{st}} A_{\mp}^{\mathrm{st}}\right)}{1+i(\widetilde{\delta}-\widetilde{\omega} / \gamma)} \\
D_{0}^{\mathrm{st}}=J-\left(A_{+}^{\mathrm{st}} B_{+}^{\mathrm{st} *}+A_{-}^{\mathrm{st}} B_{-}^{\mathrm{st} *}+\text { c.c. }\right), \\
D_{ \pm 2}^{\mathrm{st}}=-\frac{\epsilon}{\eta}\left(A_{ \pm}^{\mathrm{st}} B_{\mp}^{\mathrm{st} *}+A_{\mp}^{\mathrm{st} *} B_{ \pm}^{\mathrm{st}}\right) .
\end{gathered}
$$

Analytical solutions for these equations can be found only in the simplest situation $r=0$ and $\alpha=0$. In this limit, the two counter-propagating waves are degenerate and a bidirectional solution also exists. However, the bidirectional solution is unstable $[8,35]$. We thus focus on the unidirectional solutions $A_{+}^{\text {st }} \neq 0$ and $A_{-}^{\text {st }}=0$ without loss of generality (the counterpropagating solution can be directly obtained by replacing + with - in the final results). Using (14) in (13) and solving the resulting differential equation, we find

$$
A_{+}^{\mathrm{st}}(s)=A_{+}^{\mathrm{st}}(0) e^{i \widetilde{\omega} s+\frac{g}{1+i(\delta-\widetilde{\omega} / \gamma)} G(s)},
$$

where

$$
G(s)=\int_{0}^{s} D_{0}^{\mathrm{st}}\left(s^{\prime}\right) d s^{\prime} .
$$

We note that $D_{0}^{\mathrm{st}}=\frac{d G(s)}{d s}$, hence using (14) and (17) in (15) yields

$$
\frac{d G}{d s}=\frac{J}{1+\frac{2 g}{1+(\widetilde{\delta}-\widetilde{\omega} / \gamma)^{2}}\left|A_{+}(0)\right|^{2} e^{\frac{2 g}{1+(\widetilde{\delta}-\widetilde{\omega} / \gamma)^{2}} G(s)}} .
$$




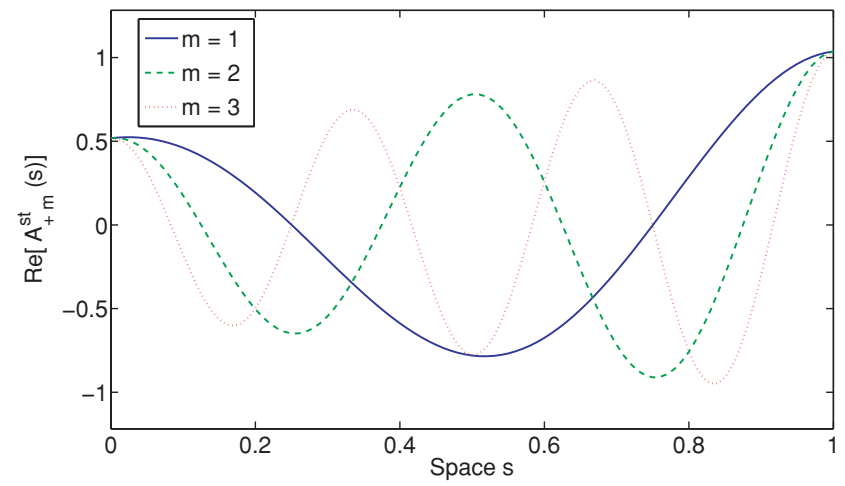

FIG. 3. (Color online) $\operatorname{Re}\left[A_{+m}^{\mathrm{st}}(s)\right]$ in the unidirectional solution. $\widetilde{\delta}=0.1, g=1, t=0.5, \gamma=100$, and $J=1.5$.

Clearly, $G(s=0)=0$, and the boundary condition for the field $A_{+}^{\text {st }}(0)=t A_{+}^{\text {st }}(1)$ imposes that

$$
\begin{gathered}
G(1)=\frac{-\ln t}{g}\left[1+(\widetilde{\delta}-\widetilde{\omega} / \gamma)^{2}\right], \\
\widetilde{\omega}=\frac{2 \pi m-\widetilde{\delta} \ln t}{1-\frac{\ln t}{\gamma}} .
\end{gathered}
$$

We note that (21) is equivalent to (9) in this simplified case. Integrating (19) from one end to the other of the laser cavity and using the boundary conditions for $G(s)$ allows us to determine

$$
\left|A_{+}^{\mathrm{st}}(0)\right|^{2}=\frac{J+\frac{\ln t}{g}\left[1+(\tilde{\delta}-\widetilde{\omega} / \gamma)^{2}\right]}{e^{-2 \ln t}-1} .
$$

We can therefore solve for $G(s)$ and determine the field profile along the laser cavity as shown in Fig. 3.

The physical insight gained in the analysis of the simplest case suggests that, in general, Eqs. (13)-(16) can be very efficiently solved by means of a numerical shooting method [36] which is useful since no analytical solution is possible in this case. In Fig. 4 a bidirectional monochromatic solution calculated in this way is shown. This shooting method can be used to quickly find the steady-state solutions for different current values, hence limited bifurcation diagrams as a function of the pump can be readily obtained. For instance,

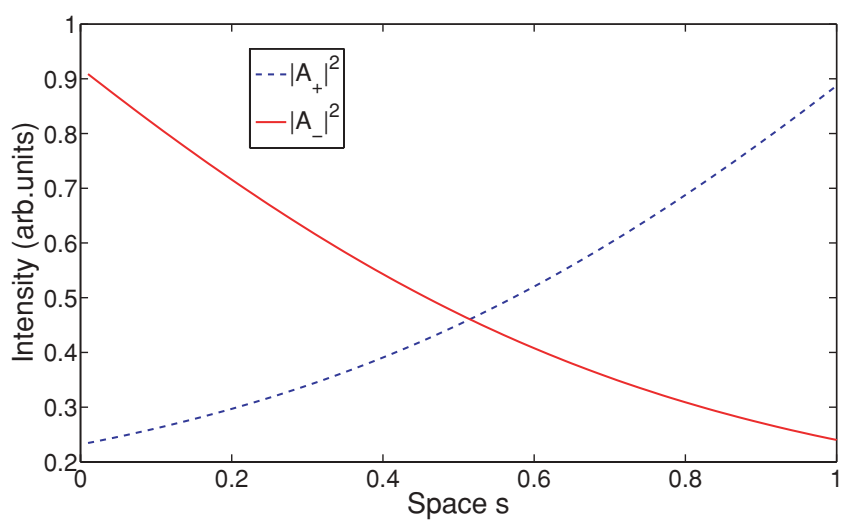

FIG. 4. (Color online) Bidirectional monochromatic solutions in the general case. Intensity of the fields inside the cavity versus space $s$. Mesh points $N=100, J=0.5, \widetilde{\delta}=0.1, g=1, \epsilon=\eta=10^{-2}$, $\beta=0, t=0.5, r=210^{-2}, \alpha=0$, and $\gamma=100$.

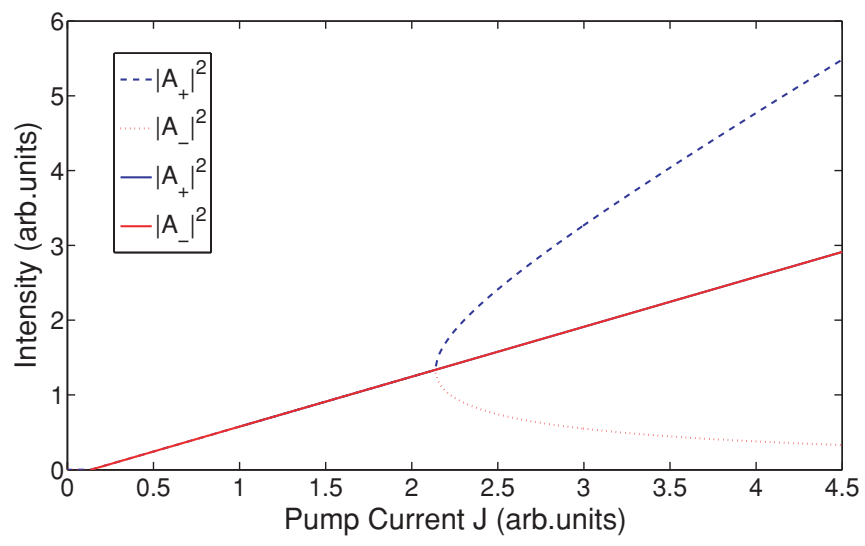

FIG. 5. (Color online) Bifurcation diagram of the monochromatic solutions: for decreasing pump $J$ showing a pitchfork bifurcation (dashed and dotted lines) and for increasing $J$ showing a bidirectional solution (solid lines). For values below $J=2.15$ all the lines coincide in the bidirectional regime. Mesh points $N=100, \widetilde{\delta}=0, g=5$, $\epsilon=10^{-2}, \eta=5, \beta=0, t=0.5, r=510^{-3}, \alpha=0$, and $\gamma=100$.

Fig. 5 depicts the pitchfork bifurcation from a bidirectional solution into two degenerate, almost unidirectional solutions that have been observed in different ring laser systems [14]. It should be noted that, in order to obtain a bifurcation diagram like that in Fig. 5, it is necessary to perform a double scan, one upward and one downward, since the shooting method follows the resulting branches even if they are unstable.

\section{TEST: SINGLE-MODE DYNAMICS}

The numerical implementation of partial-differential equations always represents a challenge from a technical point of view. In particular, the usual numerical diffusion present in most algorithms has to be carefully taken care off. While numerical dissipation can be helpful in contexts like, for example, fluid mechanics to prevent spurious solutions to rise, multimode laser dynamics is mainly governed by extremely weak gain difference between consecutive modes that correspond to increasing spatial frequencies. Any weak numerical dissipation would therefore profoundly affect the dynamical scenario and has to be avoided. To this purpose we employ a numerical algorithm that is based on the one presented in [29], which takes advantage of the fact that the equations for the electric fields can be formally solved by integration along the characteristics.

In this section we discuss the tests performed in order to check the correctness and accuracy of the numerical algorithm used to implement the TWM, which is required for controlling potential implementation mistakes. The details of the numerical implementation are described in Appendix B, where we also discuss in detail how the boundary conditions are imposed.

Clearly, the results in Secs. II A and II B provide a first test of the accuracy of the numerical implementation. We have verified that our numerical scheme accurately recovers the lasing threshold yielding monochromatic solutions that match those obtained by the shooting method. 
A further test, presented below, is provided by comparing our numerical results in the single longitudinal mode limit with the dynamical results previously obtained by Zeghlache et al. [33] with a rate equation model for a $\mathrm{CO}_{2}$ ring laser. In such a model, obtained in the good cavity limit for a pure single-longitudinal ring laser $(r=0)$, the only term that mixes the counter-propagating fields is the carrier grating, hence the bidirectional regime is unstable [8,35]. Moreover, the analysis performed in [33] demonstrates that the unidirectional solution can also become unstable in some pump and detuning regimes. For certain values of these parameters, square-wave oscillations between the counter-propagating fields appear followed by regular or even chaotic oscillations. Scanning the pump $J$ for fixed detuning, the system, which is initially stable or bistable, becomes unstable at a certain value, and it eventually recovers stability at high pump values; for fixed pump, instead, stable behavior is not recovered upon increasing detuning although it must be recalled that the single-mode approximation will eventually break down and the model in [33] be no longer valid.

A meaningful comparison of our results from those in [33] requires one to establish the equivalence among the parameters in both models. In order to do so, we reduce our model to that in [33] by neglecting any spatial dependence while redefining the losses in (1) as $\alpha_{T}=\alpha-\ln t$ (i.e., the total loss). Then, comparison with Eq. (3.11) in [33] yields the parameter correspondence rules,

$$
d_{\|}=\frac{\epsilon}{\alpha_{T}}, \quad A=\frac{g J}{\alpha_{T}}, \quad \Delta=-\widetilde{\delta} .
$$

Our numerical simulations reproduce accurately the behaviors described in [33]. We perform simulations fixing the pump and increasing the detuning (see Fig. 6); in this case, we go from the unidirectional steady emission to a region of instability where the counter-propagating fields develop a square-wave oscillation with one intensity in antiphase with the other [Fig. 6(a)]. Increasing the detuning the square waves become distorted and a secondary oscillation appears [Figs. 6(b) and 6(c)], progressing until a chaotic oscillation is obtained for high detunings as shown in Fig. 6(d). (a)

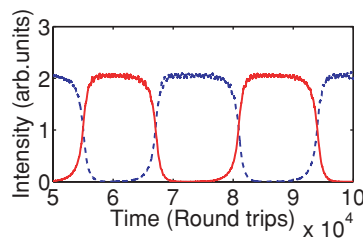

(c)

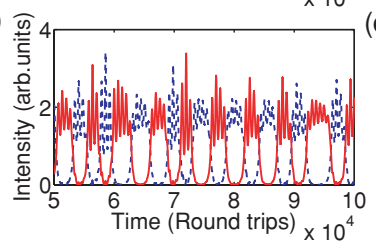

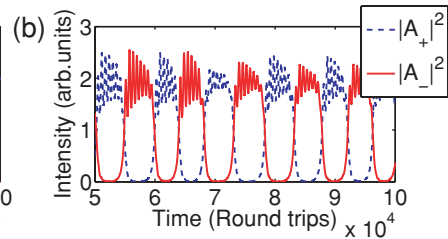

(d)

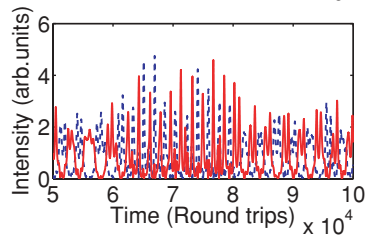

FIG. 6. (Color online) Dynamical behaviors observed for fixed pump $J=0.5$ while scanning detuning $\widetilde{\delta}$. (a) $\widetilde{\delta}=0.2$, (b) $\widetilde{\delta}=0.5$, (c) $\widetilde{\delta}=0.7$, (d) $\widetilde{\delta}=0.9$. The parameters correspond to those used in [33] in Figs. 10(a)-10(i) except for the fact that in our case the two modes have equal losses: Mesh points $N=100, g=1, \epsilon=\eta=$ $1.7810^{-5}, \beta=10^{-4}, t=0.9, r=0, \alpha=0$, and $\gamma=1$. (a)
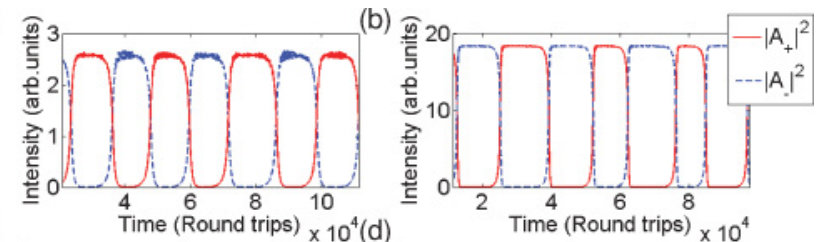

(c)
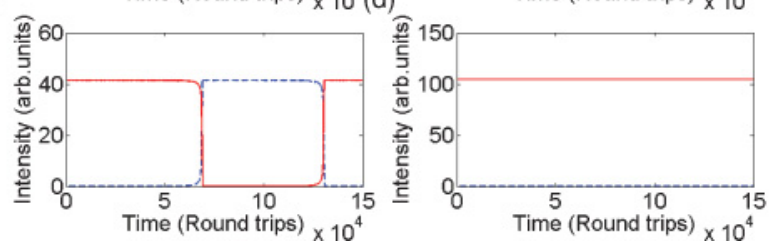

FIG. 7. (Color online) Dynamical behaviors obtained for fixed detuning $\widetilde{\delta}=0.2$ while scanning $J$. (a) $J=0.6$, (b) $J=3.6$, (c) $J=8$, (d) $J=20$ for the same parameters as in Fig. 6 .

On the other hand, when we fix the detuning and scan the pump (see Fig. 7) we pass from a unidirectional steady solution near threshold into a region of instability where square waves similar to those in the previous case developed. In contrast with the previous case, now the system recovers stability upon increasing $J$ and returns to one of the unidirectional solutions. The mechanism is a slowing of the square-wave modulation as we increase the pump [see Fig. 7(c)], a characteristic behavior of heteroclinic bifurcations.

Finally, we remark that the above behaviors are recovered even when putting a small direct reflection and spontaneous emission provided that the good cavity limit still applies (see Figs. 8 and 9); for example, they are robust against small imperfections and noise. However, if the reflectivity is too large, the system emits bidirectionally at threshold and its dynamical behavior is no longer the same $[14,16]$.

\section{MULTIMODE DYNAMICS}

The rate-equation model described in [33] is very successful at describing the rich variety of dynamics that can be encountered while in single-mode operation. However, in a real laser, increasing the detuning will eventually lead to at least a change in lasing mode which is not accounted for in the $\mathrm{RE}$ model. Indeed, the maximum allowed detuning in a real

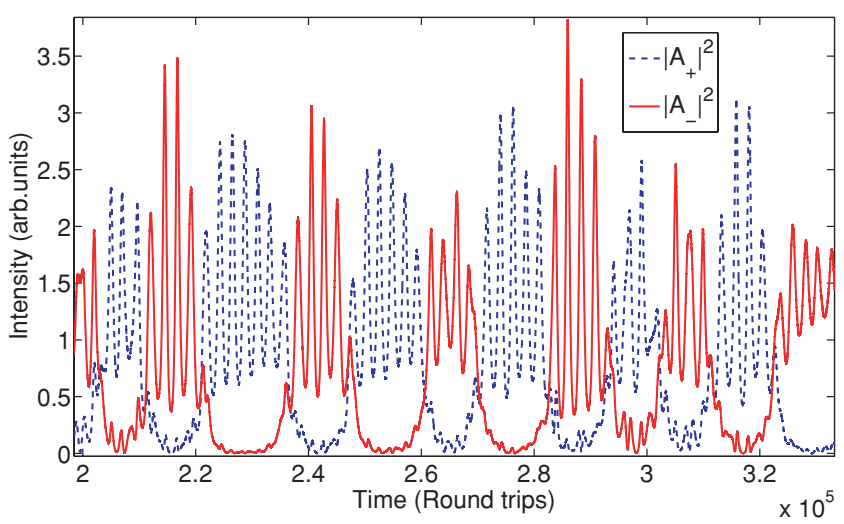

FIG. 8. (Color online) Single-mode chaotic behavior. Mesh points $N=100, J=0.4, \widetilde{\delta}=0.4, g=1, \epsilon=\eta=1.7810^{-5}, \beta=$ $10^{-4}, t=0.9, r=510^{-4}, \alpha=0$, and $\gamma=1$. 


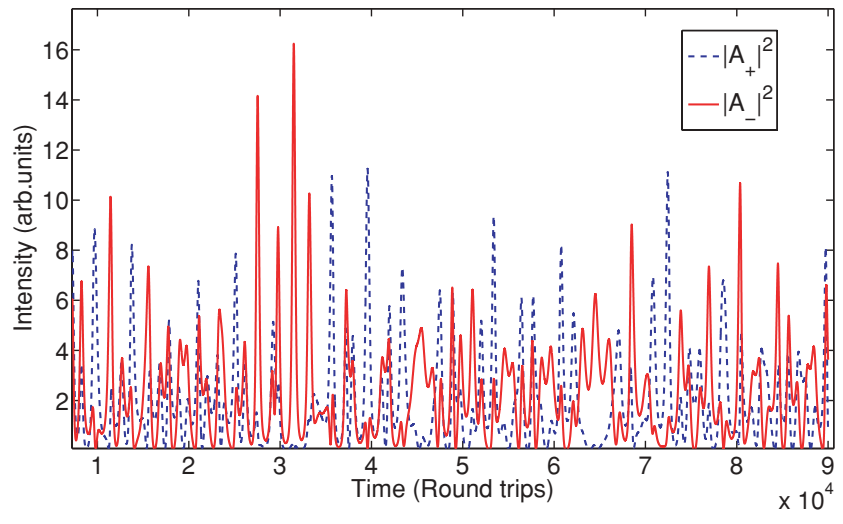

FIG. 9. (Color online) Single-mode chaotic behavior. Mesh points $N=100, J=0.5, \widetilde{\delta}=1, g=1, \epsilon=\eta=1.7810^{-5}, \beta=$ $10^{-4}, t=0.9, r=510^{-4}, \alpha=0$, and $\gamma=1$.

device corresponds to having the gain peak just between two laser modes (i.e., $\widetilde{\delta}=\pi / \gamma$ in our parametrization); in this case, the two modes will have the same threshold and lasing can be quite different than when only one mode is active. In addition, instabilities arising from the multimode character of the system as, for example, the Risken-Nummedal instability [37] can develop when the gain curve is broader than the mode spacing and the pump level is high enough.

The dynamics in these cases can readily be analyzed with the traveling wave model, which naturally retains the dynamics of the different modes and the effects of the detuning. Hence, it can allow one to explore the dynamics of the system in cases where different longitudinal modes are active.

In this section we present and discuss some remarkable dynamical behaviors obtained in these situations, although we note that the large variety of scenarios that we have observed calls for the development of a bifurcation tool of our TWM that would allow us to better understand the role played by the different parameters. To the best of our knowledge, these results have not been previously reported in the literature. It should be noted, however, that some of them are obtained for very high pumping levels, $J \sim 10-100 J_{\text {th }}$, which might be difficult or even impossible to achieve in an experiment. First, we present the situation where a moderate gain bandwidth is taken into account, and how different behaviors arise in this case depending on the pump and the detuning. In the second part of this section the case of a large gain bandwidth is discussed.

\section{A. Moderate gain bandwidth}

We consider here the case when the gain spectrum has moderate width, $\gamma=10$. We first discuss the case when the gain spectrum peak lies just between two modes, $\widetilde{\delta}=0.3141$. In this case, modes $m=0$ and $m=1$ have exactly the same threshold, so the dynamical scenario at the laser threshold corresponds to a degenerate Hopf bifurcation. It should, moreover, be noted that for each of these frequencies there are two different solution branches which for small $r$ are also almost degenerate, as discussed in Sec. II A. This highly degenerated situation allows the system to lase in a great

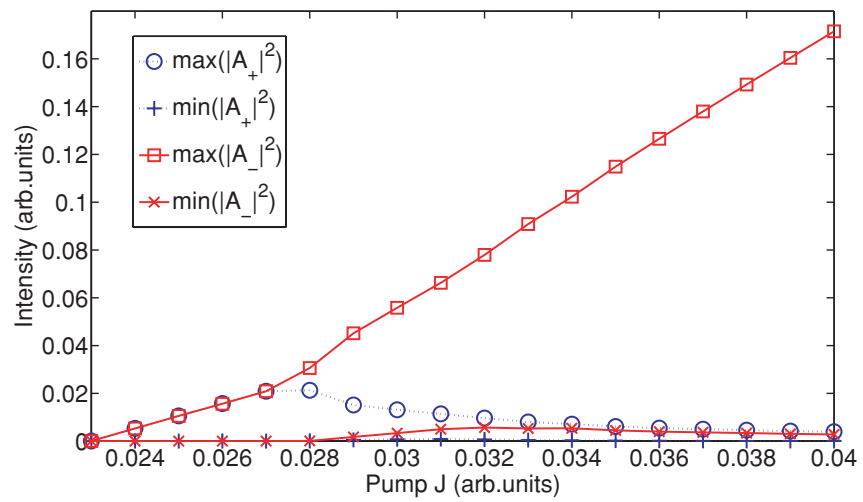

FIG. 10. (Color online) Bifurcation diagram near threshold. The fields begin to emit multimode bidirectionally, then after $J=0.028$ the backward (-) field is favored. Mesh points $N=100, \widetilde{\delta}=0.3141$, $g=5, \epsilon=10^{-2}, \eta=0.1, \beta=10^{-4}, t=0.9, r=510^{-4}, \alpha=0$, and $\gamma=10$.

variety of possible states, which can give rise to unexpected dynamical behaviors. We subsequently discuss the effect of the detuning in this case, since varying the detuning allows one to reduce the degeneracy of the system.

Figure 10 shows the bifurcation diagram near the threshold for the ring laser with moderate gain bandwidth (laser parameters specified in the caption). First, the two counterpropagating fields are both emitting with equal intensity in two modes separated by one-mode spacing (i.e., the laser starts to emit bidirectionally in consecutive modes, $m=0$ and $m=1$ ). As we increase the pump, one of the directions becomes dominant over the other, and additional modes are excited. For high enough pump (see Fig. 11), the system emits almost unidirectionally; however, the emission exhibits $100 \%$ oscillations at the round-trip time which correspond to an emission spectrum that involves four dominant modes. Further increasing the pump, the intensity oscillation becomes nonlinear, which corresponds to the locking of a moderate number of modes (see Fig. 12); this regime can be interpreted as a shallow mode-locked solution.

At even higher pumps, the nonlinear oscillation disappears and the emission becomes again bidirectional with both

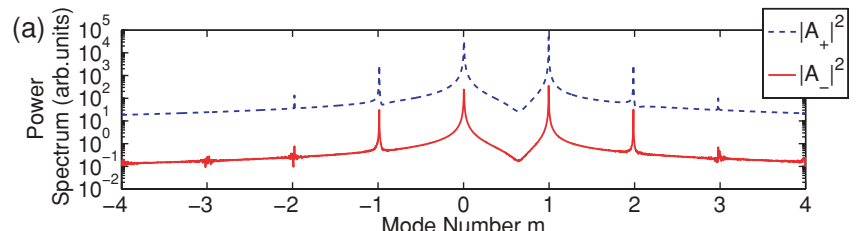

(b)

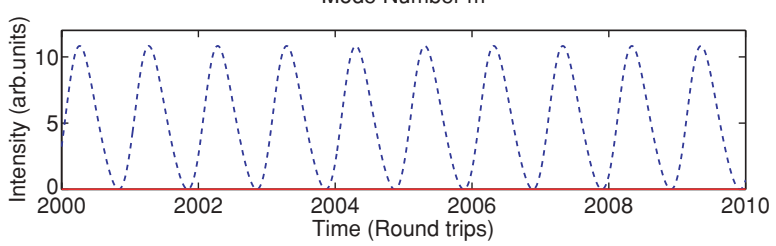

FIG. 11. (Color online) Unidirectional oscillating emission. (a) Power spectra. (b) Time trace. Mesh points $N=400, J=1, \widetilde{\delta}=$ $0.3141, g=5, \epsilon=10^{-2}, \eta=0.1, \beta=10^{-4}, t=0.9, r=510^{-4}$, $\alpha=0$, and $\gamma=10$. 


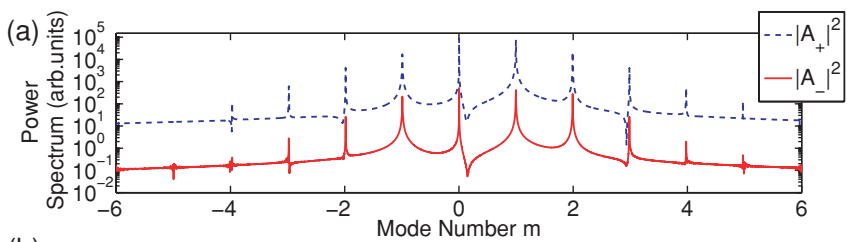

(b)

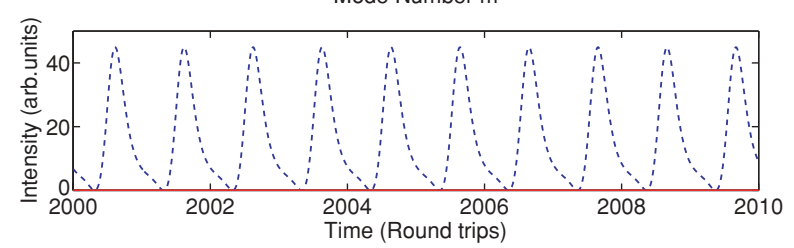

FIG. 12. (Color online) Mode-locked solution. (a) Power spectra. (b) Time trace. $J=3$. For other parameters, see Fig. 11.

directions emitting stable and with the same power (see Fig. 13).

However, a closer look at the emission in this regime (see Fig. 14) reveals that, surprisingly, each emission direction is dominated by a single mode, $m=0$ for $A_{+}$and $m=1$ for $A_{-}$. Hence each mode contributes in complementary ways to lasing in each direction: while emission in the forward direction is dominated by the reddest mode, the backward direction lases dominantly on the bluest mode. This regime is, of course, twofold degenerate.

This transition comes from the fact that the population inversion grating tries to favor the almost unidirectionnal emission at the same frequency because it induces an effective cross saturation of the gain between the forward and backward waves [8] which is larger if they have the same frequency [see Eqs. (1)-(4)]. But the power extracted from the system in the bidirectional monochromatic state is not optimal because the atoms located at the nodes of the standing wave do not contribute to stimulated emission. The power extraction can be increased in the case of bichromatic emission when the gain curve is broad enough and the frequency separation between the modes is larger than the decay rate of the population grating $(2 \pi \gg \eta)$. In this case, the population grating cannot develop in response to the counter-propagating fields,

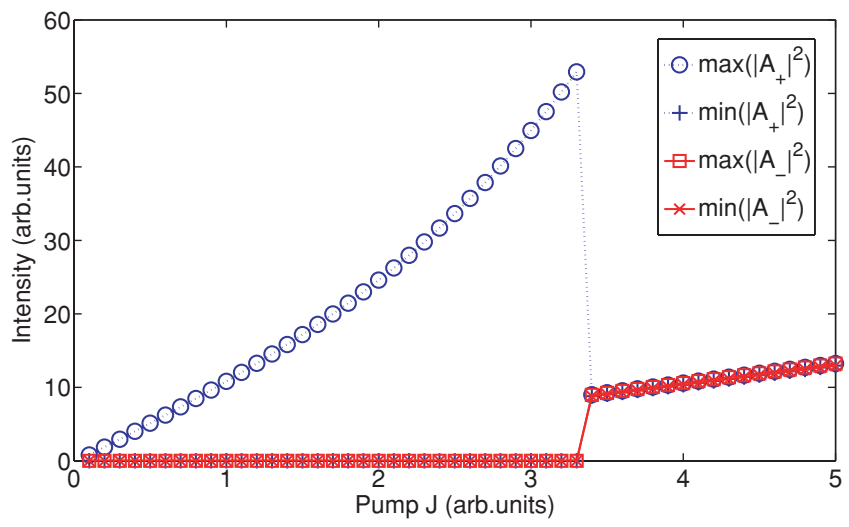

FIG. 13. (Color online) Bifurcation diagram showing the transition from unidirectional oscillating emission to bidirectional emission at different frequencies. (a) Power spectra. (b) Time trace. Mesh points $N=400, \widetilde{\delta}=0.3141, g=5, \epsilon=10^{-2}, \eta=0.1, \beta=10^{-4}, t=0.9$, $r=510^{-4}, \alpha=0$, and $\gamma=10$.

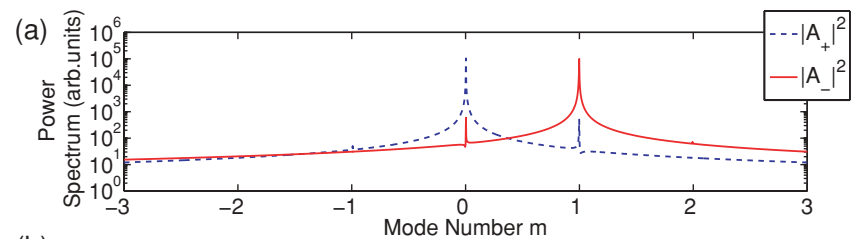

(b)

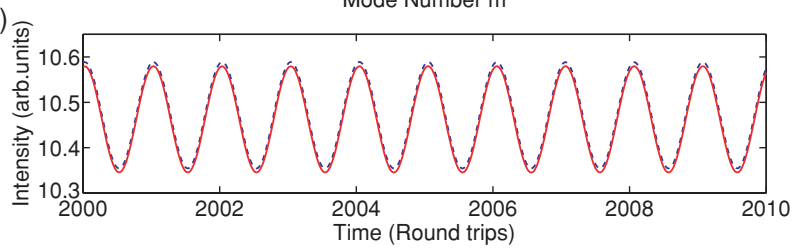

FIG. 14. (Color online) Bidirectional oscillating emission at different frequencies. (a) Power spectra. (b) Time trace. $J=4$. For other parameters, see Fig. 11.

hereby effectively reducing cross-gain saturation between the forward and backward waves and restoring the possibility of obtaining stable bidirectional operation. We conclude that this is a pure dynamical effect that allows for bichromatic bidirectional emission at high current.

\section{Dependence on detuning}

In order to see the effect of the detuning on the behavior of the laser in the case of moderate gain bandwidth, we perform simulations for different $\widetilde{\delta}$. For $\delta=0.3$, the laser begins to emit bidirectionally in a mode $m=0$ but it rapidly becomes almost unidirectional with a small amplitude oscillation that corresponds to residual emission in mode $m=1$ (see inset in Fig. 15). As we increase the pump, the emission becomes increasingly unidirectional and single mode until $J=0.4$, where mode $m=1$ starts to lase and favors the opposite direction. Above this pump value, the laser emits bidirectionally with each direction dominated by a different mode as in the previous subsection. However, the nonsymmetrical position of the cavity modes with respect to the peak of the gain curve

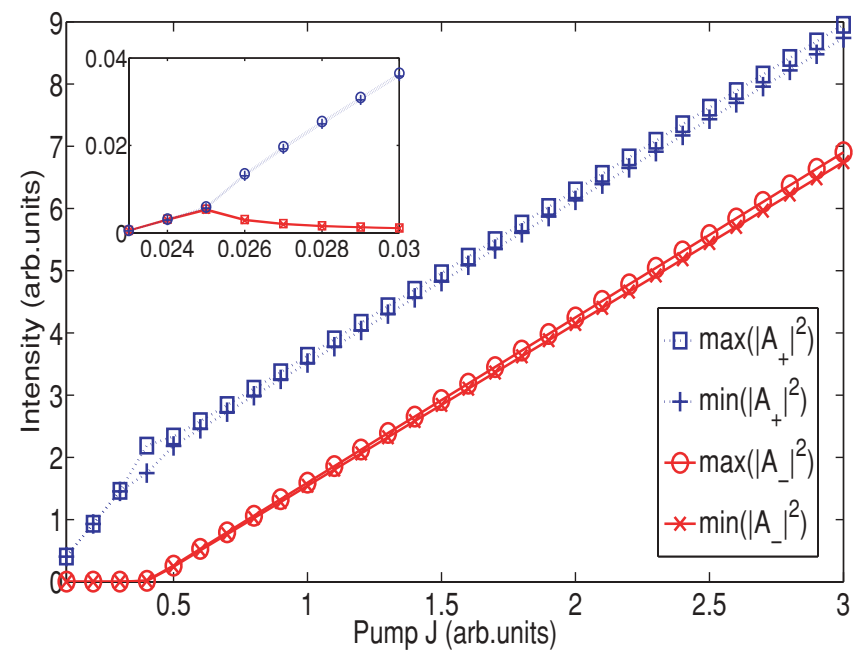

FIG. 15. (Color online) Bifurcation diagram for $\gamma=10$ and $\tilde{\delta}=$ 0.3 . (Inset) Bifurcation diagram near the threshold. Mesh points $N=$ $400, g=5, \epsilon=10^{-2}, \eta=0.1, \beta=10^{-4}, t=0.9, r=510^{-4}$, and $\alpha=0$. 
(a)

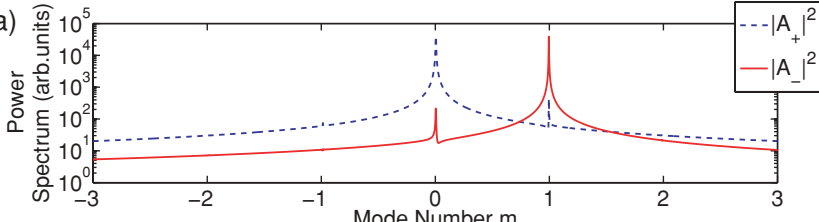

(b)

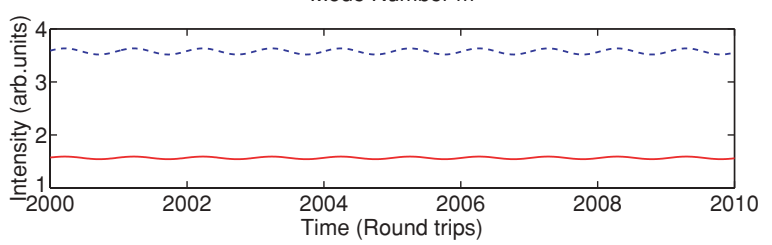

FIG. 16. (Color online) Bidirectional emission. (a) Power spectra. (b) Time trace. Mesh points $N=400, J=1, \widetilde{\delta}=0.3, g=5, \epsilon=$ $10^{-2}, \eta=0.1, \beta=10^{-4}, t=0.9, r=510^{-4}, \alpha=0$, and $\gamma=10$.

produces a sensible difference between the intensities of the two counter-propagating fields (see Fig. 16).

The above results have been obtained by starting the simulations from a noisy initial condition that does not favor any of the emission directions. However, when the simulations are launched from an initial condition that privileges one of the directions (see Fig. 17), we find for some current values an almost unidirectional solution oscillating at the modal beat note with almost $100 \%$ amplitude. This solution is analogous to that in Fig. 13 in the previous subsection, and it eventually also disappears into the bidirectional solution of Fig. 15 . The former result evidences that the unidirectional oscillating solution and the bidirectional emission at different frequencies can coexist depending on the parameters. We have tried to induce jumps among these two types of solutions by injecting optical pulses, but we have not managed to stably control the emission state of the system; after a relatively long transient, the system returned to the original emission state, indicating that in spite of their coexistence, the perturbation in phase space requires specific characteristics to place the system into the basin of attraction of the other solution.

Finally, in the case that the gain peak is close to one of the cavity modes, multimode dynamics is suppressed because the mode closest to the gain peak takes all the energy provided to the system. For a detuning value $\widetilde{\delta}=0.15$ the laser emits single mode unidirectionally as shown in Fig. 18.

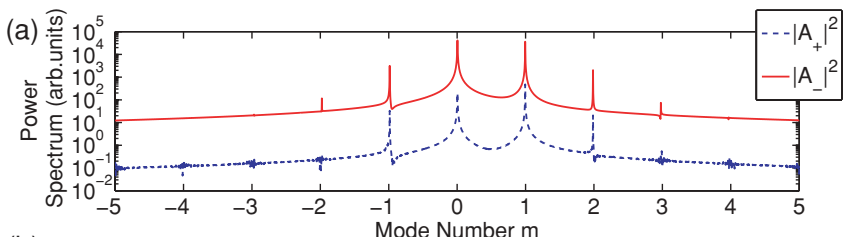

(b)

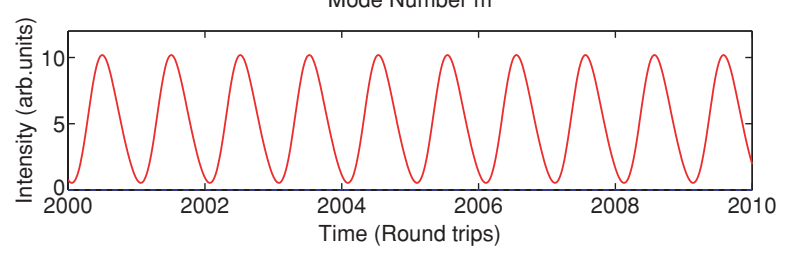

FIG. 17. (Color online) Unidirectional oscillating emission. (a) Power spectra. (b) Time trace. For parameters, see Fig. 16.

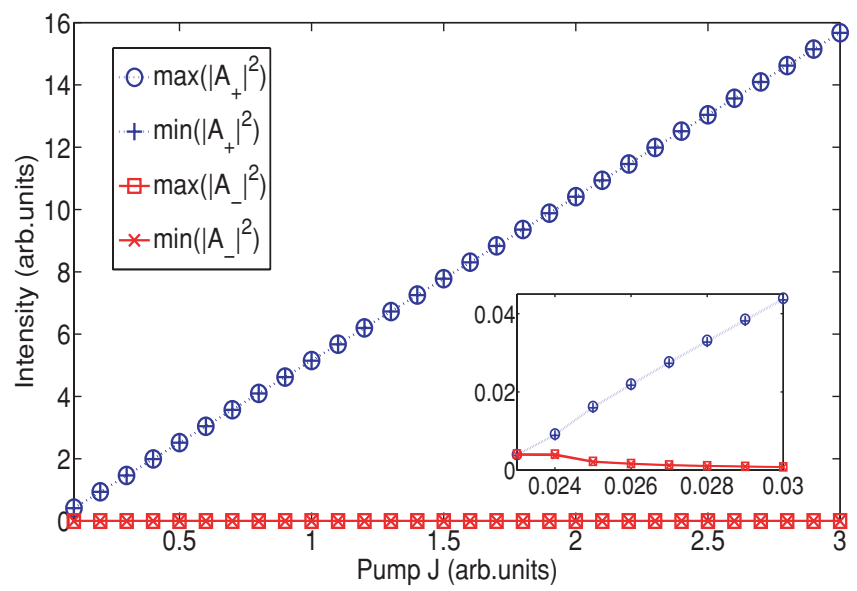

FIG. 18. (Color online) Bifurcation diagram for $\gamma=10$ and $\tilde{\delta}=$ 0.15. (Inset) Bifurcation diagram near the threshold. Mesh points $N=400, g=5, \epsilon=10^{-2}, \eta=0.1, \beta=10^{-4}, t=0.9, r=510^{-4}$, and $\alpha=0$.

\section{B. Large gain bandwidth}

In this section we consider a large gain bandwidth $(\gamma=$ 100) that allows for a rich variety of dynamical behaviors because a large number of modes can become active.

The bifurcation diagram shown in Fig. 19 summarizes the different behaviors observed when the peak of the gain curve is just between the first two modes, $\widetilde{\delta}=0.03141$. Close to threshold, the laser emits bidirectionally with two modes active in each direction as in Fig. 10. Increasing the pump, the forward direction becomes dominant and mode $m=0$ dominates; conversely, the backward direction is

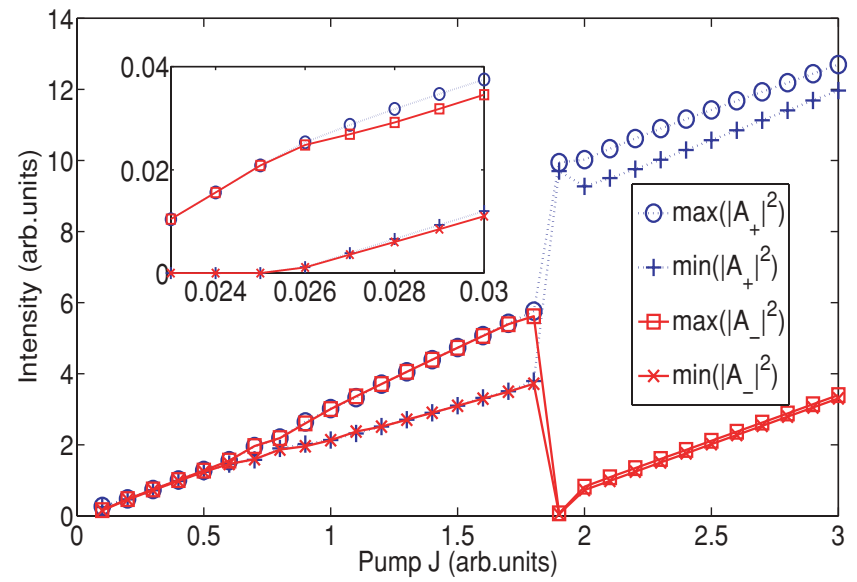

FIG. 19. (Color online) Bifurcation diagram showing different behaviors for $\gamma=100$ and $\widetilde{\delta}=0.03141$. (Inset) Bifurcation diagram near the threshold. First, close to threshold, the laser emits bidirectionally with both counter-propagating fields emitting at two consecutive modes. Then a regime of bidirectional emission at different frequencies appears (see Fig. 20). Third, an oscillating regime where the counter-propagating fields are out of phase (see Fig. 21). Fourth, a unidirectional multimode solution, composed by not-consecutive modes (see Fig. 22). Fifth, a bidirectional emission at different frequencies at not-consecutive modes (see Fig. 23). Mesh points $N=400, g=5, \epsilon=10^{-2}, \eta=210^{-2}, \beta=10^{-4}, t=0.9$, $r=510^{-4}$, and $\alpha=0$. 


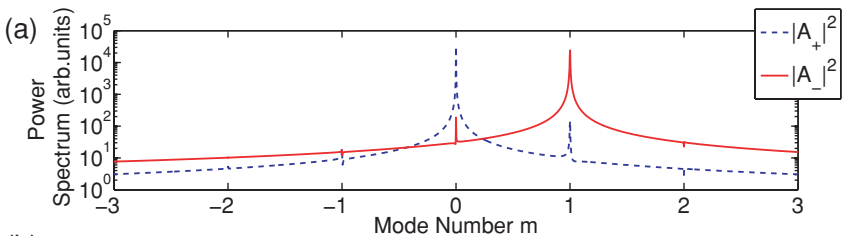

(b)

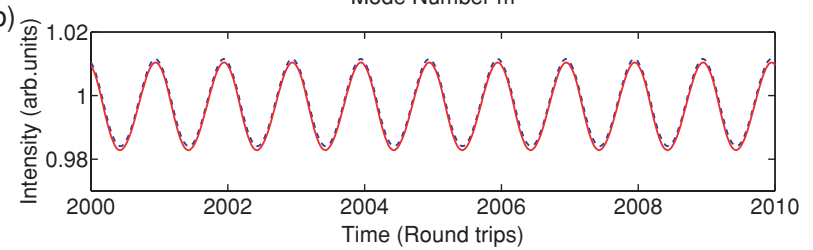

FIG. 20. (Color online) Bidirectional emission at different frequencies. (a) Power spectra. (b) Time trace. Mesh points $N=400$, $J=0.4, \widetilde{\delta}=0.03141, g=5, \epsilon=10^{-2}, \eta=210^{-2}, \beta=10^{-4}, t=$ $0.9, r=510^{-4}, \alpha=0$, and $\gamma=100$.

dominated by mode $m=1$ (see Fig. 20). In this regime, both emission directions oscillate in phase, but as the pump is still increased, more modes become excited and the oscillations of the intensity of the counter-propagating fields are out of phase (see Fig. 21). Still increasing the pump, a regime of almost single-mode, unidirectional emission is recovered (see Fig. 22) for a small range of pump values. We see that in this case, the depressed emission direction is dominated by mode $m=2$, with a secondary peak on mode $m=-2$ excited by four-wave mixing processes. Such a regime indicates that the gain suppression of mode $m=1$ by emission on mode $m=0$ is strong enough to inhibit emission on mode $m=1$. However, the large bandwidth of the gain curve allows modes farther away from mode $m=0$ to become active when the pump is still increased. As shown in Fig. 23, this leads again to a bidirectional solution where each direction dominantly lases on different modes separated by twice the mode spacing.

\section{Dependence on detuning}

For detuning values above $\widetilde{\delta}=0.025$, the behavior of the system is qualitatively the same described in the previous subsection (see Fig. 24). However, the nonsymmetrical position of the gain curve peak makes the DC component of the fields different and a unidirectional solution is found near threshold.

(a)

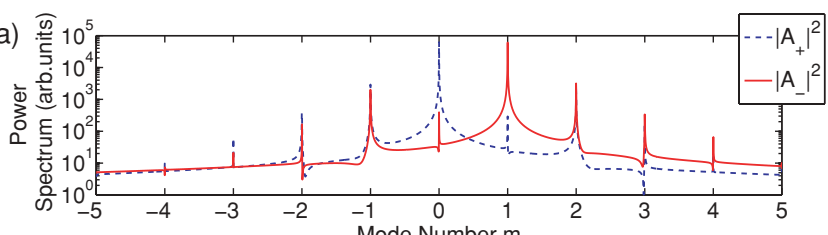

(b)

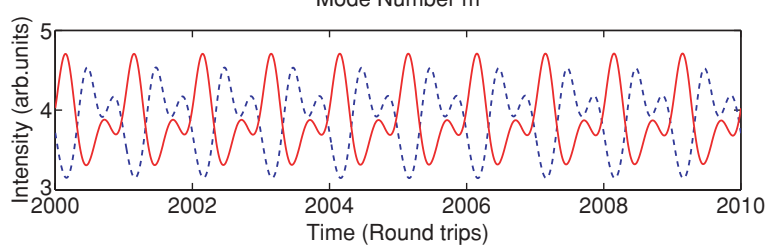

FIG. 21. (Color online) Multimode alternate oscillations. (a) Power spectra. (b) Time trace. $J=1.5$. Other parameters are as in Fig. 20.

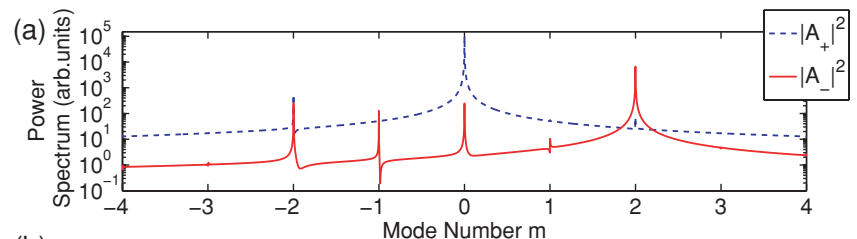

(b)

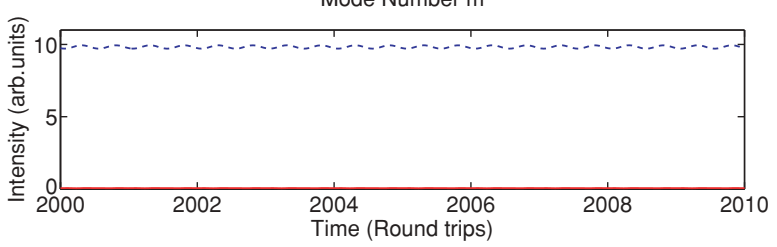

FIG. 22. (Color online) Unidirectional emission. (a) Power spectra. (b) Time trace. $J=1.9$. Other parameters are as in Fig. 20.

(a)

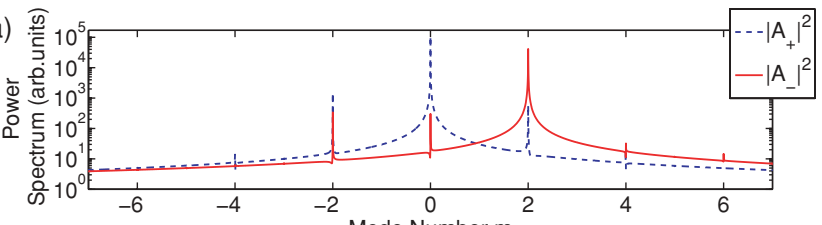

(b)

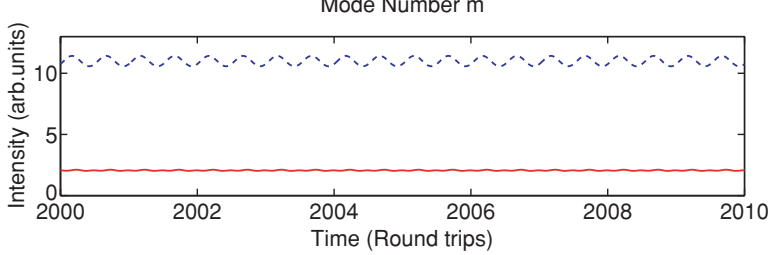

FIG. 23. (Color online) Bidirectional emission at different frequencies. (a) Power spectra. (b) Time trace. $J=2.5$. Other parameters are as in Fig. 20.

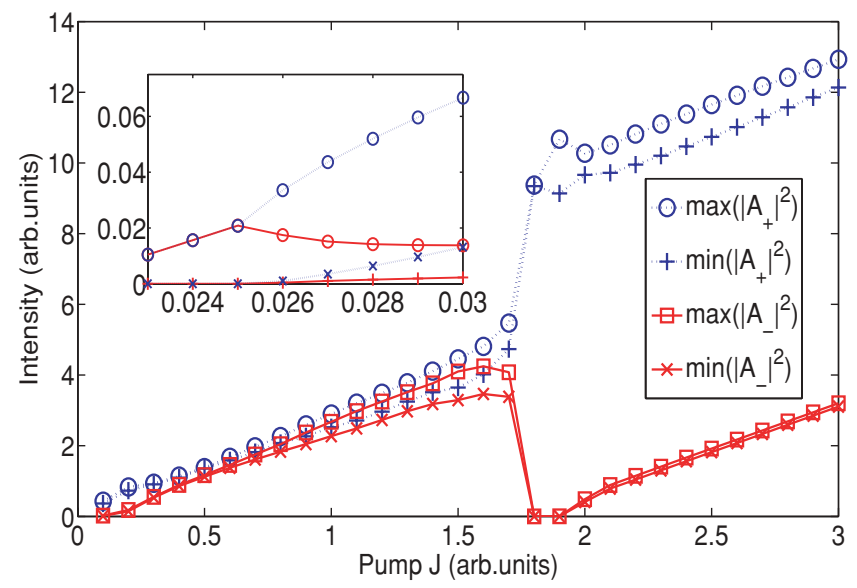

FIG. 24. (Color online) Bifurcation diagram for $\gamma=100$ and $\widetilde{\delta}=0.03$. (Inset) Bifurcation diagram near the threshold. First, bidirectional emission is found near threshold, then one of the fields is suppressed and a unidirectional regime is found; after that the suppressed field begins to emit at a different frequency with respect to that emitted by the not- suppressed field and a bidirectional solution appears. Increasing the pump we find a unidirectional solution that ends up in a bidirectional solution emitting at not-consecutive modes. Mesh points $N=400, g=5, \epsilon=10^{-2}, \eta=210^{-2}, \beta=10^{-4}$, $t=0.9, r=510^{-4}$, and $\alpha=0$. 


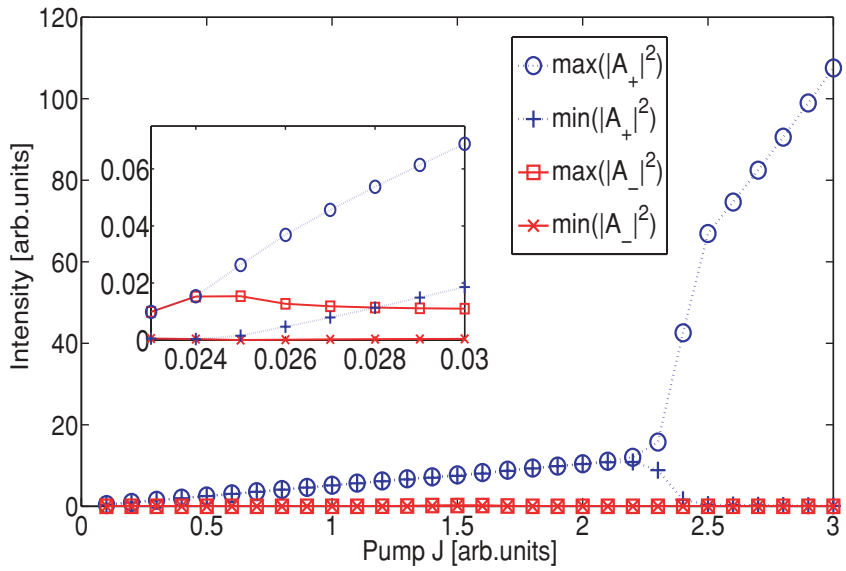

FIG. 25. (Color online) Bifurcation diagram for $\gamma=100$ and $\widetilde{\delta}=$ 0.015. (Inset) Bifurcation diagram near the threshold. Near threshold the laser emits bidirectionally but for a wide range on pump the laser emits unidirectionally single mode, then near $J=2.2$ different modes start to lase, and a mode-locked solution arises (see Fig. 26). Mesh points $N=400, g=5, \epsilon=10^{-2}, \eta=210^{-2}, \beta=10^{-4}, t=0.9$, $r=510^{-4}$, and $\alpha=0$.

A different scenario emerges at low detunings. When the detuning is decreased to a value $\widetilde{\delta}=0.015$ (see Fig. 25), the laser starts emitting bidirectionally with both directions emitting on two consecutive modes. For slightly higher pump, one emission direction starts to dominate with quasi-singlemode emission up to $J \approx 2.4$, where a unidirectional solution arises with a high number of active modes (see Fig. 26). Although this solution appears very far away from the lasing threshold, it is worth being examined in detail. The solution has the characteristics of a unidirectional mode-locked state, since the laser emits sharp and narrow pulses being in one direction only. Note that this is a harmonic mode-locked state, with pulses occurring at twice the fundamental repetition rate. The duty cycle of the pulses is around 6\%. It is worth remarking that this solution appears without inserting in the cavity any additional element that favors pulsed operation (i.e., a saturable absorber or alike), but it merely arises from an instability of the $\mathrm{cw}$ solution occurring when the power level is such that the Rabi frequency of the two-level atoms equals the

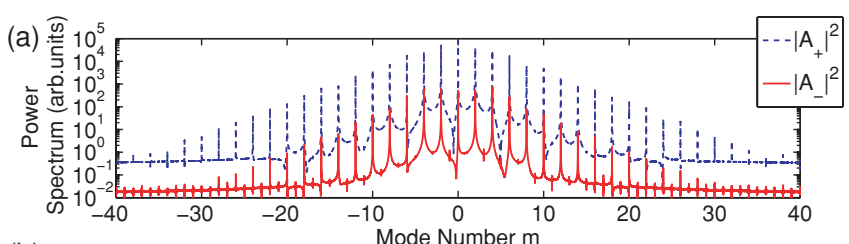

(b)

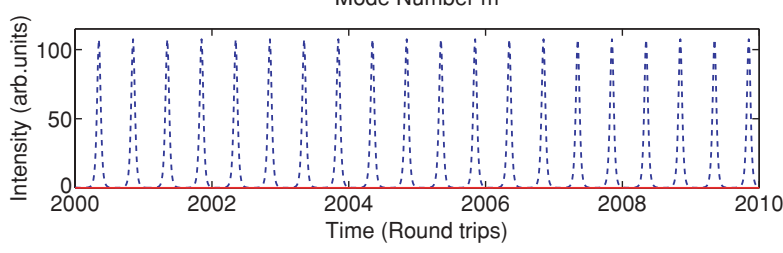

FIG. 26. (Color online) Mode-locked emission. (a) Power spectra. (b) Time trace. Mesh points $N=400, J=3, \widetilde{\delta}=0.015, g=5$, $\epsilon=10^{-2}, \eta=210^{-2}, \beta=10^{-4}, t=0.9, r=510^{-4}, \alpha=0, \gamma=$ $100, n=1$ and $L=1 \mathrm{~m}$. polarization dephasing rate. From this point of view, then, the mechanism that triggers this solution is analogous to that in the Risken-Nummedal instability. The main difference between our case and the classical Risken-Nummedal instability is that the large gain curve that we are considering allows for the excitation of additional side modes through four-wave mixing processes mediated by both $D_{0}$ and $D_{ \pm 2}$, which give rise to the pulsed emission of the system.

\section{CONCLUSIONS}

The multimode dynamics of a two-level ring laser has been explored using a bidirectional TWM. The model and its numerical implementation have been tested by reproducing the dynamical results obtained in the single-mode limit by Zeghlache et al. [33]. We have shown that the dynamical regimes reported in [33] are robust against noise and residual reflections provided that the single-mode limit holds. We have found dynamical regimes where the emission in each direction occurs at different wavelengths, each direction being associated with a different longitudinal mode. In addition, the influence of the detuning and the width of the gain spectrum have been thoroughly analyzed, and the onset of unidirectional, mode-locked emission for large gain bandwidth and relatively small detuning has been studied in detail.

\section{ACKNOWLEDGMENTS}

The authors thank A. Scirè for helpful discussion. A. P. thanks the Department of Electronics and Electrical Engineering of the University of Glasgow for its hospitality where part of this work was developed. This work was partially funded by the european project IOLOS IST-2005-34743. A. P. acknowledges financial support from the Govern Balear. J. J. acknowledges financial support from the EPSRC (Project No. EP/E065112/1). S. B. acknowledges financial support from project BLANCO (Project No. PN TEC2006-13887C05-03).

\section{APPENDIX A: DERIVATION OF THE MODEL}

The wave equation for an electric field $\mathcal{E}(z, t)$ in a medium can be written as

$$
\frac{\partial^{2} \mathcal{E}}{\partial z^{2}}-\frac{n^{2}}{c^{2}} \frac{\partial^{2} \mathcal{E}}{\partial t^{2}}=\mu \frac{\partial^{2} \mathcal{P}}{\partial t^{2}},
$$

where $\mathcal{P}$ is the polarization of the medium, $n$ is the refraction index of the medium, $c$ is the speed of light in vacuum, and $\mu$ is the magnetic permeability of the medium $[31,38]$. Assuming a quasimonochromatic field around the optical carrier frequency $\omega_{0}$, we can express it as

$$
\mathcal{E}(z, t)=F(z, t) e^{-i \omega_{0} t}+\text { c.c. },
$$

where c.c. denotes complex conjugate and $F(z, t)$ is the slowly time-varying amplitude of the field, that is, $|\partial F / \partial t|$ $\ll \omega_{0}|F|$.

Analogously, the polarization of the active medium can be decomposed as

$$
\mathcal{P}(z, t)=i\left[\Pi(z, t) e^{-i \omega_{0} t}-\text { c.c. }\right],
$$


where $\Pi(z, t)$ is the slowly time-varying amplitude of the material polarization. Assuming a quasiresonant two-level medium-composed of $N_{A}$ identical two-level atoms per unit volume with a resonance frequency $\omega_{A}$ between the upper and the lower levels - the polarization $\Pi$ can be determined, in the rotating wave approximation, by the density matrix formalism $[31,38]$. Its time evolution is given by

$$
\frac{\partial \Pi}{\partial t}=-\left(i \delta+\gamma_{\perp}\right) \Pi-\frac{\tilde{\mu}^{2}}{\hbar} N F,
$$

where $N(z, t)$ is the population inversion density, $\delta$ is the detuning, which takes into account the difference between the atomic transition frequency and the emission frequency (i.e., $\left.\delta=\omega_{A}-\omega_{0}\right), \gamma_{\perp}$ is the polarization decay rate, and $\tilde{\mu}$ is the component of the dipole moment along the direction of the field. $N$ in turn evolves according to

$$
\frac{\partial N}{\partial t}=\frac{2}{\hbar}\left(F \Pi^{*}+\text { c.c. }\right)+\mathrm{J}-\gamma_{\|} \mathrm{N}+\mathbb{D} \partial_{\mathrm{z}}^{2} \mathrm{~N},
$$

where $Q$ is the external pump, $\hbar$ is the Planck's constant divided by $2 \pi, \gamma_{\|}$is the population inversion decay rate, and $\mathbb{D}$ is the diffusion coefficient $[31,38]$.

Noting that amplification of the field usually requires propagation over distances long compared to the optical wavelength $\lambda_{0}=2 \pi c /\left(n \omega_{0}\right)$, we can further decompose the local field amplitude into forward $(+)$ and backward (-) propagating components,

$$
F(z, t)=E_{+}(z, t) e^{i q_{0} z}+E_{-}(z, t) e^{-i q_{0} z},
$$

where $E_{ \pm}(z, t)$ are slowly varying both in space and time, as compared with $q_{0}^{-1}$ and $\omega_{0}^{-1}$, respectively. Analogously, we have that

$$
\Pi(z, t)=P_{+}(z, t) e^{i q_{0} z}+P_{-}(z, t) e^{-i q_{0} z} .
$$

Using these decompositions we can write the slowly varying approximation (SVA) [31] for the wave equation (A1) as

$$
\pm \frac{\partial E_{ \pm}}{\partial z}+\frac{n}{c} \frac{\partial E_{ \pm}}{\partial t}=-\frac{\omega_{0} c \mu}{2 n} P_{ \pm} .
$$

These equations are complemented with boundary conditions [39] for the geometry considered, in this case a ring, that can be written as

$$
\begin{gathered}
E_{+}(0)=t_{+} E_{+}(L) e^{i q_{0} L}+r_{-} E_{-}(0), \\
E_{-}(L) e^{-i q_{0} L}=t_{-} E_{-}(0)+r_{+} E_{+}(L) e^{i q_{0} L},
\end{gathered}
$$

where $L$ is the length of the ring and $t_{ \pm}$and $r_{ \pm}$denote the transmissivity and reflectivity of the forward and backward waves, which follows $\left|t_{ \pm}\right|^{2}+\left|r_{ \pm}\right|^{2}=1-\varepsilon_{ \pm}$, where $\varepsilon_{ \pm}$are the losses at the point coupler.

Using (A6) and (A7) into (A4) and (A5) evidences that the presence of counter-propagating fields creates a spatial modulation of the population inversion. This important property follows from the iterative relationship between the diagonal and off-diagonal matrix elements of the density matrix $\hat{\rho}$ [40]. As a result, only odd harmonics appear in the expansion of $\mathcal{P}$ and only even harmonics appear in the expansion of the population difference $D$. This spatial modulation acts as a Bragg grating and creates a coupling between the counterpropagating fields. In order to get the dynamics of this grating explicitly we decompose the population difference in different spatial contributions as

$$
N=N_{0}+N_{+2} e^{2 i q_{0} z}+N_{-2} e^{-2 i q_{0} z}+\cdots
$$

Such a decomposition yields an infinite hierarchy of equations that has to be truncated in order to keep the problem treatable. In systems with large diffusion, the truncation can be justified due to the quadratically increasing damping of the high-order terms [30,32]; in other cases, the intensity of the fields has to be low compared to the saturation intensity of the medium [33]. To the dominant order, the medium evolves according to

$$
\begin{gathered}
\frac{\partial P_{ \pm}}{\partial t}=-\left(i \delta+\gamma_{\perp}\right) P_{ \pm}-\frac{\tilde{\mu}^{2}}{\hbar}\left(N_{0} E_{ \pm}+N_{ \pm 2} E_{\mp}\right), \\
\frac{\partial N_{0}}{\partial t}=\frac{2}{\hbar}\left(E_{+} P_{+}^{*}+E_{-} P_{-}^{*}+\text { c.c. }\right)+J-\gamma_{\|} N_{0}+\mathbb{D} \partial_{z}^{2} N_{0},
\end{gathered}
$$

and

$$
\frac{\partial N_{ \pm 2}}{\partial t}=\frac{2}{\hbar}\left(E_{ \pm} P_{\mp}^{*}+E_{\mp}^{*} P_{ \pm}\right)-\left(\gamma_{\|}+4 q_{0}^{2} \mathbb{D}\right) N_{ \pm 2},
$$

where we have used twice that $\left|\partial_{z} N_{ \pm 2}\right| \ll q_{0}\left|N_{ \pm 2}\right|$. We can see in that diffusion tries to smear out the grating in the population inversion (A13) by inducing a much larger effective relaxation rate for $N_{ \pm 2}$ than for $N_{0}$. We can also see in (A11) that the polarization in the forward direction has a contribution from the field in the backward direction and vice versa. This "reflection on the grating" leads to cross-saturation of the fields, as could be seen by adiabatic elimination of $P_{ \pm}$.

Equations (A8) and (A11)-(A13) are equivalent to Eq. (3.4) in [33] with the only difference that we retained the slow spatial dependence of the fields while the good cavity limit was invoked in [33] in order to work with time-dependent field amplitudes only. In this way, our approach allows for describing multimode dynamics that is beyond the scope of [33].

For numerical purposes it is convenient to rewrite Eqs. (A8) and (A11)-(A13) in dimensionless form,

$$
\begin{gathered}
\pm \frac{\partial A_{ \pm}}{\partial s}+\frac{\partial A_{ \pm}}{\partial \tau}=B_{ \pm}-\alpha A_{ \pm} \\
\frac{1}{\gamma} \frac{\partial B_{ \pm}}{\partial \tau}=-(1+i \tilde{\delta}) B_{ \pm}+g\left(D_{0} A_{ \pm}+D_{ \pm 2} A_{\mp}\right) \\
+\sqrt{\beta D_{0}} \xi_{ \pm}(s, \tau) \\
\frac{1}{\epsilon} \frac{\partial D_{0}}{\partial \tau}=J-D_{0}+\Delta \frac{\partial^{2} D_{0}}{\partial s^{2}}-\left(A_{+} B_{+}^{*}+A_{-} B_{-}^{*}+\text { c.c. }\right) \\
\frac{1}{\eta} \frac{\partial D_{ \pm 2}}{\partial \tau}=-D_{ \pm 2}-\frac{\epsilon}{\eta}\left(A_{ \pm} B_{\mp}^{*}+A_{\mp}^{*} B_{ \pm}\right)
\end{gathered}
$$

where $D_{0}=N_{0} / N_{A}, D_{ \pm 2}=N_{ \pm 2} / N_{A}$ and we have scaled the fields and polarizations as

$$
A_{ \pm}=\sqrt{\frac{4 n}{\mu \omega_{0} c \hbar \gamma_{\|} L}} E_{ \pm}, \quad B_{ \pm}=-\sqrt{\frac{\mu \omega_{0} c}{n \hbar \gamma_{\|} L}} P_{ \pm} .
$$


We have also included in Eq. (A14) a term modeling the internal losses $\alpha$ for the electric fields, we have defined new dimensionless parameters,

$$
\begin{gathered}
g=\frac{\mu \omega_{0} c \tilde{\mu}^{2} L}{2 n \hbar \gamma_{\perp}}, \quad \gamma=\frac{\gamma_{\perp} n L}{c}, \quad \epsilon=\frac{\gamma_{\|} n L}{c}, \\
\eta=\frac{\left(\gamma_{\|}+4 q_{0}^{2} \mathbb{D}\right) n L}{c}, \quad \Delta=\frac{\mathbb{D}}{\gamma_{\|} L^{2}}, \quad \tilde{\delta}=\frac{\delta}{\gamma_{\perp}},
\end{gathered}
$$

and finally we have defined new coordinates,

$$
\tau=\frac{c}{n L} t, \quad s=\frac{z}{L} .
$$

In this new reference frame, the general boundary conditions for the fields in the laser read

$$
\begin{gathered}
A_{+}(0)=t_{+} A_{+}(1) e^{i \gamma \widetilde{\omega}_{0}}+r_{-} A_{-}(0), \\
A_{-}(1) e^{-i \gamma \widetilde{\omega}_{0}}=t_{-} A_{-}(0)+r_{+} A_{+}(1) e^{i \gamma \widetilde{\omega}_{0}} .
\end{gathered}
$$

Usually we shall take $\gamma \widetilde{\omega}_{0}=2 \pi m$ where $m=0, \pm 1, \pm 2 \ldots$ then $e^{i \gamma \widetilde{\omega}_{0}}=1$ without loss of generality. It means that we take the carrier frequency $\omega_{0}$ as one of the modes of the cavity.

We note that the effects of diffusion in (A16) are almost negligible because the characteristic length scale of $D_{0}$ is 1 (i.e., the cavity length), so that we can set $\Delta=0$ in (A16). Instead, we should retain it in (A17) because the characteristic length scale in this case is the emission wavelength $\lambda_{0}=$ $2 \pi c / \omega_{0}$.

Finally, spontaneous emission is modeled by including Langevin noise terms $\xi_{ \pm}(s, \tau)$ [30]. They are taken to be Gaussian white noise in space and time with zero mean and correlations $\left\langle\xi_{ \pm}(s, \tau) \xi_{ \pm}\left(s^{\prime}, \tau^{\prime}\right)\right\rangle=\delta\left(\tau-\tau^{\prime}\right) \delta\left(s-s^{\prime}\right)$, and their intensities are proportional to the population density [41].

\section{APPENDIX B: NUMERICAL ALGORITHM}

The numerical algorithm used to perform the simulation of the normalized system of Eqs. (1)-(4) is based in the one presented in [29]. This algorithm takes advantage of the fact that the equations for the fields can be solved formally in terms of integrals of the polarizations. We discretize time with time step $h$, hence the spatial grid has also discretization step $h$. All spatial points $n=1, \ldots, N$ are internal, with the first and last

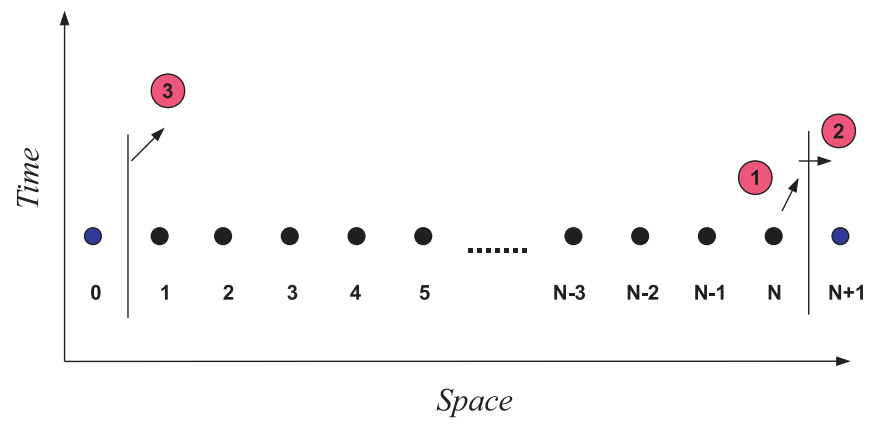

FIG. 27. (Color online) Schematic representation of spatial discretization and the implementation of the boundary conditions for the $A_{+}$electric field. In three steps: 1) Half-step explicit Euler; 2) boundary conditions; 3) Half-step implicit Euler. The mesh is composed by $N$ points and $N$ intervals and two auxiliary points at 0 and $N+1$ added for the implementation. points located at $h / 2$ from the nearest end (see Fig. 27). We denote by $X_{j}^{n}$ the value of variable $X$ at time $t=n h$ and grid point $s=j h$.

We use the midpoint discretization scheme for the fields [29], so they are updated according to

$$
\begin{aligned}
& A_{+j}^{n+1}=\frac{1-q}{1+q} A_{+j-1}^{n}+p\left(B_{+j-1}^{n}+B_{+j}^{n+1}\right), \\
& A_{-j}^{n+1}=\frac{1-q}{1+q} A_{-j+1}^{n}+p\left(B_{-j+1}^{n}+B_{-j}^{n+1}\right),
\end{aligned}
$$

where $q=\alpha h / 2, p=(h / 2)(1+q)^{-1}$. For the polarizations we have

$$
\begin{aligned}
B_{ \pm j}^{n+1}= & \mu B_{ \pm j}^{n}+v D_{0 j}^{n+1 / 2}\left(A_{ \pm j}^{n+1}+A_{ \pm j}^{n}\right) \\
& +v D_{ \pm 2 j}^{n+1 / 2}\left(A_{\mp j}^{n+1}+A_{\mp j}^{n}\right)+\sqrt{\beta h D_{0}} \xi_{ \pm},
\end{aligned}
$$

where $\mu=[1-(\gamma h / 2)(1+i \widetilde{\delta})][1+(\gamma h / 2)(1+i \widetilde{\delta})]^{-1}$ and $v=(g h \gamma / 2)[1+(\gamma h / 2)(1+i \tilde{\delta})]^{-1}$, and where we have used the approximation,

$$
\int_{t}^{t+\Delta t} D_{k}(t) A_{l}(t) d t \simeq \Delta t D_{k}\left(t+\frac{\Delta t}{2}\right) \frac{A_{l}(t+\Delta t)+A_{l}(t)}{2} .
$$

At this point we note that Eq. (B3) needs the values of the carriers $\left(D_{0}\right.$ and $\left.D_{ \pm 2}\right)$ at intermediate time steps, hence we use a temporal grid for the carrier densities which is staggered by half a time step from the fields and polarizations. This is different from the original algorithm in [29], where the carriers are on the same temporal grid than the fields and the polarizations and then interpolation is used to evaluate the carriers at the intermediate times needed in (B1)-(B3). In our case, the finite difference equations for carriers are, thus,

$$
\begin{aligned}
D_{0 j}^{n+3 / 2}= & \rho D_{0 j}^{n+1 / 2}+\theta J \\
& -\theta\left(A_{+j}^{n+1} B_{+j}^{* n+1}+A_{-j}^{n+1} B_{-j}^{* n+1}+\text { c.c. }\right) \\
D_{ \pm 2 j}^{n+3 / 2}= & \rho D_{ \pm 2 j}^{n+1 / 2}, \quad-\theta\left(A_{ \pm j}^{n+1} B_{\mp j}^{* n+1}+A_{\mp j}^{* n+1} B_{ \pm j}^{n+1}\right),
\end{aligned}
$$

where $\rho=\left(1+\frac{\epsilon h}{2}\right)\left(1-\frac{\epsilon h}{2}\right)^{-1}$ and $\theta=\epsilon h\left(1-\frac{\epsilon h}{2}\right)^{-1}$.

\section{Boundary conditions}

In order to impose the general boundary conditions (5), we have to consider that the fields propagate during half a step, then experience partial reflection and transmission and then they propagate for another half a step. In addition, we recall the ring structure of our system, hence points $j=1$ and $j=N$ are connected through the boundary conditions. This procedure for $A_{+}$and $A_{-}$is implemented as follows:

Step 1. We use an explicit Euler method to compute the value of the fields just before arriving at boundary by propagating the fields over half a step,

$$
\begin{gathered}
A_{+N+1 / 2}^{n+1 / 2}-(1-q) A_{+N}^{n}=\frac{h}{2} B_{+N}^{n}, \\
A_{-1 / 2}^{n+1 / 2}-(1-q) A_{-1}^{n}=\frac{h}{2} B_{-1}^{n} .
\end{gathered}
$$


Step 2. We apply the boundary conditions and compute the fields just after the boundary, which are denoted as $\widetilde{A}_{+}$and $\widetilde{A}_{-}$,

$$
\begin{gathered}
\widetilde{A}_{+1 / 2}^{n+1 / 2}=t_{+} A_{+N+1 / 2}^{n+1 / 2}+r_{-} A_{-1 / 2}^{n+1 / 2}, \\
\widetilde{A}_{-N+1 / 2}^{n+1 / 2}=t_{-} A_{-1 / 2}^{n+1 / 2}+r_{+} A_{+N+1 / 2}^{n+1 / 2} .
\end{gathered}
$$

Step 3. Finally we use the implicit Euler method for the remaining half a step to calculate the value of the fields at time $n+1$,

$$
\begin{aligned}
(1+q) A_{+1}^{n+1}-\widetilde{A}_{+1 / 2}^{n+1 / 2} & =\frac{h}{2} B_{+1}^{n+1} \\
(1+q) A_{-N}^{n+1}-\widetilde{A}_{-N+1 / 2}^{n+1 / 2} & =\frac{h}{2} B_{-N}^{n+1} .
\end{aligned}
$$

Note that these procedures can be very efficiently implemented by adding to the spatial grid two auxiliary points $j=0$ and $j=N+1$ (see Fig. 27) located half a step away from the facets where the fields and polarizations are

$$
\begin{gathered}
A_{+0}^{n}=t_{+} A_{+N}^{n}+r_{-} A_{-1}^{n} \\
B_{+0}^{n}=t_{+} B_{+N}^{n}+r_{-} B_{-1}^{n} \\
A_{-N+1}^{n}=t_{-} A_{-1}^{n}+r_{+} A_{+N}^{n} \\
B_{-N+1}^{n}=t_{-} B_{-1}^{n}+r_{+} B_{+N}^{n},
\end{gathered}
$$

and updating the fields by means of the standard midpoint integration,

$$
\begin{gathered}
A_{+1}^{n+1}=\frac{1-q}{1+q} A_{+0}^{n}+p\left(B_{+0}^{n}+B_{+1}^{n+1}\right), \\
A_{-N}^{n+1}=\frac{1-q}{1+q} A_{-N+1}^{n}+p\left(B_{-N+1}^{n}+B_{-N}^{n+1}\right) .
\end{gathered}
$$

[1] H. W. Schröder, L. Stein, D. Frölich, B. Fugger, and H. Welling, Appl. Phys. 14, 377 (1977).

[2] M. R. Surette, L. Goldberg, and D. Mehuys, IEEE Photonics Technol. Lett. 5, 919 (1993).

[3] X. Donga, H. Tama, B. Guana, C. Zhaoa, and X. Dong, Opt. Commun. 224, 295 (2003).

[4] S. V. Zhukovsky, D. N. Chigrin, and J. Kroha, Phys. Rev. A 79, 033803 (2009).

[5] S. L. McCall, A. F. J. Levi, R. E. Slusher, S. J. Pearten, and R. A. Logan, Appl. Phys. Lett. 60, 289 (1992).

[6] V. M. Apalkov and M. E. Raikh, Phys. Rev. B 70, 195317 (2004).

[7] W. W. Chow, J. Gea-Banacloche, L. M. Pedrotti, V. E. Sanders, W. Schleich, and M. O. Scully, Rev. Mod. Phys. 57, 61 (1985).

[8] M. Sargent III, Phys. Rev. A 48, 717 (1993).

[9] M. T. Hill, H. J. S. Dorren, T. de Vrie, X. J. M. Leijtens, J. H. den Besten, B. Smalbrugge, Y. S. Oei, H. Binsma, G. D. Khoe, and M. K. Smit, Nature 432, 206 (2004).

[10] W. E. Lamb Jr., Phys. Rev. 134, A1429 (1964).

[11] F. Aronowitz, Phys. Rev. 139, A635 (1965).

[12] L. N. Menegozzi and W. E. Lamb Jr., Phys. Rev. A 8, 2103 (1973).

[13] M. Sorel, A. Scirè, G. Giuliani, R. Miglierina, S. Balle, P. J. R. Laybourn, and S. Donati, Opt. Lett. 27, 1992 (2002).

[14] M. Sorel, G. Giuliani, A. Scirè, R. Miglierina, S. Donati, and P. J. R. Laybourn, IEEE J. Quantum Electron. 39, 1187 (2003).

[15] M. Sorel, P. J. R. Laybourn, G. Giuliani, and S. Donati, Appl. Phys. Lett. 80, 3051 (2002).

[16] C. Born, M. Sorel, and S. Yu, IEEE J. Quantum Electron. 41, 261 (2005).

[17] H. Nakatsuka, S. Asaka, H. Itoh, K. Ikeda, and M. Matsuoka, Phys. Rev. Lett. 50, 109 (1983).

[18] D. G. Rabus, Z. Bian, and A. Shakouri, IEEE J. Sel. Top. Quantum Electron. 13, 1249 (2007).

[19] M. Choi, T. Tanaka, S. Sunada, and T. Harayama, Appl. Phys. Lett. 94, 231110 (2009).

[20] H. Yanagi, R. Takeaki, S. Tomita, A. Ishizumi, F. Sasaki, K. Yamashita, and K. Oe, Appl. Phys. Lett. 95, 033306 (2009).

[21] H. T. Hattori, C. Seassal, E. Touraille, P. Rojo-Romeo, X. Letartre, G. Hollinger, P. Viktorovitch, L. Di Cioccio,
M. Zussy, L. El Melhaoui, and J. M. Fedeli, IEEE Photonics Technol. Lett. 18, 223 (2006).

[22] R. D. Kekatpure and M. L. Brongersma, Phys. Rev. A 78, 023829 (2008).

[23] D. Simeonov, E. Feltin, A. Altoukhov, A. Castiglia, J.-F. Carlin, R. Butté, and N. Grandjean, Appl. Phys. Lett. 92, 171102 (2008).

[24] S. Koseki, B. Zhang, K. De Greve, and Y. Yamamoto, Appl. Phys. Lett. 94, 051110 (2009).

[25] I. Lo, C. Hsieh, Y. Hsu, W. Pang, and M. Chou, Appl. Phys. Lett. 94, 062105 (2009).

[26] W. R. Christian and L. Mandel, Phys. Rev. A 34, 3932 (1986).

[27] G. Vemuri and R. Roy, Phys. Rev. A 39, 4668 (1989).

[28] C. Etrich, P. Mandel, R. Centeno Neelen, R. J. C. Spreeuw, and J. P. Woerdman, Phys. Rev. A 46, 525 (1992).

[29] J. A. Fleck Jr., Phys. Rev. B 1, 84 (1970).

[30] M. Homar, J. V. Moloney, and M. San Miguel, IEEE J. Quantum Electron. 32, 553 (1996)

[31] L. M. Narducci and N. B. Abraham, Laser Physics and Laser Instabilities (World Scientific, Singapore, 1988).

[32] J. Javaloyes and S. Balle, IEEE J. Quantum Electron. 45, 431 (2009).

[33] H. Zeghlache, P. Mandel, N. B. Abraham, L. M. Hoffer, G. L. Lippi, and T. Mello, Phys. Rev. A 37, 470 (1988).

[34] S. Fürst, A. Pérez-Serrano, A. Scirè, M. Sorel, and S. Balle, Appl. Phys. Lett. 93, 251109 (2008).

[35] P. Mandel and G. P. Agrawal, Opt. Commun. 42, 269 (1982).

[36] W. H. Press, S. A. Teukolsky, W. T. Vetterling, and B. P. Flannery, Numerical Recipes, The Art of Scientific Computing, 3rd ed. (Cambridge University Press, New York, 2007).

[37] H. Risken and H. Nummedal, J. Appl. Phys. 39, 4662 (1968).

[38] A. Yariv, Quantum Electronics (John Wiley \& Sons, New York, 1989).

[39] A. E. Siegman, Lasers (University Science Books, Mill Valley, 1986).

[40] S. Haroche and F. Hartmann, Phys. Rev. A 6, 1280 (1972).

[41] K. Petermann, Laser Diode Modulation and Noise (Kluwer Academic Publishers, Norwell, 1988). 